\title{
Towards quantitative electrostatic potential mapping of working semiconductor devices using off-axis electron holography
}

Yazdi, Sadegh; Kasama, Takeshi; Beleggia, Marco; Samaie Yekta, Maryam; McComb, David W.; Twitchett-Harrison, Alison C.; Dunin-Borkowski, Rafal E.

\section{Published in:}

Ultramicroscopy

Link to article, DOI:

10.1016/j.ultramic.2014.12.012

Publication date:

2015

Document Version

Peer reviewed version

Link back to DTU Orbit

Citation (APA):

Yazdi, S., Kasama, T., Beleggia, M., Samaie Yekta, M., McComb, D. W., Twitchett-Harrison, A. C., \& DuninBorkowski, R. E. (2015). Towards quantitative electrostatic potential mapping of working semiconductor devices using off-axis electron holography. Ultramicroscopy, 152, 10-20. https://doi.org/10.1016/j.ultramic.2014.12.012

\section{General rights}

Copyright and moral rights for the publications made accessible in the public portal are retained by the authors and/or other copyright owners and it is a condition of accessing publications that users recognise and abide by the legal requirements associated with these rights.

- Users may download and print one copy of any publication from the public portal for the purpose of private study or research.

- You may not further distribute the material or use it for any profit-making activity or commercial gain

- You may freely distribute the URL identifying the publication in the public portal 


\section{Towards quantitative electrostatic potential mapping of working}

2 semiconductor devices using off-axis electron holography

3 Sadegh Yazdi ${ }^{1,2}$, Takeshi Kasama ${ }^{2}$, Marco Beleggia ${ }^{2}$, Maryam Samaie Yekta ${ }^{2}$, David W. McComb ${ }^{1,3}$,

4 Alison C. Twitchett-Harrison ${ }^{1}$ and Rafal E. Dunin-Borkowski ${ }^{2,4}$

$5 \quad{ }^{1}$ Department of Materials, Imperial College London, London SW7 2AZ, United Kingdom

$6 \quad{ }^{2}$ Center for Electron Nanoscopy, Technical University of Denmark, DK 2800 Lyngby, Denmark

$7 \quad{ }^{3}$ Department of Materials Science and Engineering, The Ohio State University, Columbus, Ohio $8 \quad 43210$, United States

$9{ }^{4}$ Ernst Ruska-Centre for Microscopy and Spectroscopy with Electrons and Peter Grünberg Institute, 10 Forschungszentrum Jülich, D-52425 Jülich, Germany

Abstract

Pronounced improvements in the understanding of semiconductor device performance are expected if electrostatic potential distributions can be measured quantitatively and reliably under working conditions with sufficient sensitivity and spatial resolution. Here, we employ off-axis electron holography to characterize an electrically-biased $\mathrm{Si} p$ - $n$ junction by measuring its electrostatic potential, electric field and charge density distributions under working conditions. A comparison between experimental electron holographic phase images and images obtained using threedimensional electrostatic potential simulations highlights several remaining challenges to quantitative analysis. Our results illustrate how the determination of reliable potential distributions from phase images of electrically biased devices requires electrostatic fringing fields, surface charges, specimen preparation damage and the effects of limited spatial resolution to be taken into account. 
24 As semiconductor devices continue to shrink, so variations in the placement of small numbers of dopant atoms can drastically change electrostatic potential distributions in the devices' active regions and affect their electrical and optical properties ${ }^{1}$. Existing methods for introducing dopants are not yet sufficiently controllable, while electrostatic potential measurement techniques are not yet precise enough for the development and understanding of future device generations ${ }^{2}$. Comprehensive feedback from a quantitative potential measurement technique is crucial for parameter optimization in device modeling, ideally in three dimensions with high spatial resolution and high precision.

The technique of off-axis electron holography in the transmission electron microscope (TEM) promises to achieve the required spatial resolution and precision for potential measurement in two dimensions $^{3}$ and can be combined with electron tomography for three-dimensional measurements ${ }^{4}$. Moreover, by carrying out electron holography on a semiconductor device that is electrically biased in situ in the TEM, it is in principle possible to map the electrostatic potential distribution of a device under working conditions, thereby providing additional information for the optimization of device design and fabrication.

An off-axis electron hologram is an interference pattern created by overlapping part of the electron wave that has passed unperturbed through vacuum (the "reference wave") with another part of the electron wave that has passed through the sample (the "object wave"). The resulting interference pattern encodes the phase difference between the reference and object waves, which can then be reconstructed, e.g., with a standard FFT-based algorithm ${ }^{5}$. Provided that the reference beam is not perturbed by the presence of the specimen, that the specimen is not magnetic and that the effect of dynamical diffraction on the phase shift is negligible, the reconstructed phase difference can be written in the form:

$$
\varphi(x, y)=C_{E} \int_{-\infty}^{+\infty} V(x, y, z) d z
$$

where $C_{E}$ is a constant that depends on the energy of the electron beam $\left(C_{E}=8.56 \mathrm{mrad} \mathrm{V}^{-1} \mathrm{~nm}^{-1}\right.$ at $120 \mathrm{keV}), V$ is the electrostatic potential and $z$ is the electron beam direction. If the electrostatic potential distribution is constant in the electron beam direction (i.e., it has no $z$-dependence) and 
limited to the interior of the specimen (i.e., there are no fringing fields), then Eq. (1) can be simplified to:

$\varphi(x, y)=C_{E} V(x, y) t(x, y)$

51

52

where $t$ is the specimen thickness. Therefore, if the specimen thickness $t(x, y)$ is known and the phase shift $\varphi(x, y)$ is measured using electron holography, then in principle the electrostatic potential distribution across the specimen can be mapped. However, despite the fact that the phase shift can be measured with high sensitivity (better than $1 \mathrm{mrad}^{6}$ ) using electron holography, the interpretation of phase images in terms of electrostatic potential distributions requires several factors to be taken into account.

As the phase shift is highly sensitive to specimen thickness, any small thickness variations can be misinterpreted as electrostatic potential variations. For example, a $3 \mathrm{~nm}$ step in thickness (e.g., due to preferential milling) in a Si specimen of thickness $300 \mathrm{~nm}$ can be misinterpreted as a built-in potential difference of $0.12 \mathrm{~V}$ (at $120 \mathrm{kV}$ accelerating voltage). A possible workaround to bypass this problem and to avoid possible misinterpretation is to electrically bias the specimen, since, to a first approximation, the phase variation across a $p-n$ junction changes with applied voltage, whereas the contribution to the phase shift due to specimen thickness variations remains unchanged.

Changes in mean inner potential (MIP) across heterojunctions must also be taken into account. Steps in phase across heterojunctions measured using electron holography depend on both the difference in MIP and the dopant potential profile across each junction, as well as on any local redistribution of charge that may be present at each interface in the sample. Differences in MIP can therefore be misinterpreted as dopant potentials, or vice versa. Because MIPs are unchanged by external voltages, it should again be possible to avoid such misinterpretation by measuring phase steps across heterojunctions under different electrical biasing conditions. Similarly, the effects of diffraction contrast on the phase shift can be misinterpreted as changes in dopant potential and can be removed by electrical biasing, so long as the contribution to the phase from diffraction contrast is unaffected by the applied electrical bias. 
The measured potential may also be affected by electrical charging of the specimen in the presence of electron beam irradiation due to the emission of secondary electrons and the generation of electronhole pairs in the specimen. The presence of electrical contacts close to the region of interest is expected to help to restore any charge imbalance resulting from secondary electron emission from the specimen $^{7}$. Electrical contacts can also be used to measure electron beam induced current (EBIC) ${ }^{8}$ and, in this way, to provide information about electron-hole pair generation.

The fact that dopant potentials are, in general, much smaller than mean inner potentials, means that a measurement with $0.1 \mathrm{~V}$ sensitivity in $\mathrm{Si}$, which has a mean inner potential of $\sim 12 \mathrm{~V}$, requires a signal to background ratio of better than $1 \%$ (to measure a $0.1 \mathrm{~V}$ dopant potential on a $12 \mathrm{~V}$ background). The ability of electron holography to detect variations in dopant potential can therefore be improved by the application of an applied electrical bias.

For all of these reasons, in situ electrical biasing of semiconductor devices in the TEM is expected to provide a valuable solution to many of the issues that need to be overcome when converting electron holographic phase images into electrostatic potential maps, as well as providing an opportunity to characterize semiconductor devices under working conditions.

Previous electron holography studies of electrically biased $p-n$ junctions have shown only qualitative agreement between experimental results and theory ${ }^{9-12}$. For example, researchers at the University of Bologna demonstrated electrical leakage fields (fringing fields) from an electrically biased $p-n$ junction into vacuum, as expected on the basis of electrostatics ${ }^{10}$. They also showed that the fringing fields increase in magnitude with applied reverse bias ${ }^{13}$. Subsequently, scientists from Cambridge demonstrated a linear increase of the step in phase across a Si $p-n$ junction with applied reverse bias in a focused ion beam (FIB) prepared specimen ${ }^{14}$.

Despite qualitative agreement between experiment and theory, the quantitative interpretation of such experimental results reveals large discrepancies. Measured fringing fields are considerably smaller than expected ${ }^{15}$, while electrostatic potentials, electric fields, charge densities and dopant concentrations inferred from phase images are almost always significantly lower than predicted values. In addition, measured charge densities across $p$ - $n$ junctions have been reported to depend on 
applied bias, while theory predicts that their magnitude should remain constant, as the depletion layer width increases with applied reverse bias ${ }^{16}$. Although electron beam irradiation and specimen preparation damage have been blamed for these discrepancies in the literature ${ }^{17,18}$, their origin is not yet fully understood. Here, we address these issues quantitatively by measuring the electrostatic potential, electric field and charge density across a Si $p$ - $n$ junction from electron holograms acquired under different electrical biasing conditions and by comparing the measurements with simulations.

\section{2) Experiment}

\section{1) Experimental Details}

An abrupt symmetrical Si $p$ - $n$ junction comprising a 4- $\mu$ m-thick As-doped (n-type) layer grown epitaxially onto a (100) oriented B-doped ( $p$-type) substrate using molecular beam epitaxy was provided by OKMETIC ${ }^{19}$. The electrically active dopant concentration was determined using a fourpoint-probe measurement to be $6 \times 10^{18} \mathrm{~cm}^{-3}$ on each side of the junction, which corresponds to an expected built-in potential of $1.02 \mathrm{~V}$ across the junction. In order to electrically bias the $p$ - $n$ junction in situ in the TEM, a $1.5 \mathrm{~mm} \times 1.5 \mathrm{~mm} \times 100 \mu \mathrm{m}$ cleaved piece of the wafer was clamped between two electrical contacts in a cartridge-based single tilt biasing holder ${ }^{20}$. A parallel-sided electron transparent membrane was then micromachined at one corner of the cleaved wedge using a $30 \mathrm{keV}$ focused ion beam (FIB) ${ }^{21}$. The length of the electron transparent membrane was kept as short as $1 \mu \mathrm{m}$, while the rest of the specimen was significantly thicker, to minimize charging during the holography observation. At the final stage of specimen preparation, at a thickness of approximately $600 \mathrm{~nm}$, low $\mathrm{keV}$ cleaning was carried out using $2 \mathrm{keV}$ FIB milling to reduce the effects of specimen surface damage and Ga implantation. The crystalline thickness of the membrane was determined to be $550 \pm 10 \mathrm{~nm}$ using convergent beam electron diffraction (CBED) in a two beam condition. The applied voltage across the junction was varied between 0 and $2 \mathrm{~V}$ reverse bias in intervals of $0.2 \mathrm{~V}$. At each voltage, both an object off-axis electron hologram and a vacuum reference electron hologram were recorded. The holograms were acquired in an FEI Titan 80-300 TEM operated at $120 \mathrm{kV}$ in Lorentz mode. By setting the biprism voltage to $70 \mathrm{~V}$ and the magnification to $18600 \times$, holograms 
with a visible overlap region of $\sim 0.6 \times 2 \mu \mathrm{m}^{2}$ at 6 pixels per fringe could be acquired on a $2 \mathrm{k} \times 2 \mathrm{k}$ charge-coupled device (CCD) camera, with a holographic interference fringe spacing of $4.6 \mathrm{~nm}$ and fringe visibility of $20 \%$ for an acquisition time of $16 \mathrm{~s}$. The $p-n$ junction was oriented exactly edge-on with respect to the electron beam by tilting the specimen to the central line of the 040 Kikuchi band, at a specimen tilt angle of $5.2^{\circ}$ from the $<001>$ zone axis.

\section{2) Experimental Results}

Representative reconstructed phase and amplitude images, obtained by applying a mask of radius 1/14 $\mathrm{nm}^{-1}$ to the sideband in the Fourier transform of the hologram for $0 \mathrm{~V}$ applied bias, are shown in Figs. 1(a) and (b), respectively. The $p$ - and $n$-regions are clearly visible in the phase image. No diffraction contrast can be seen in the amplitude image at this specimen orientation, suggesting that dynamical diffraction does not affect the phase step across the $p$ - $n$ junction significantly.

The step in phase across the junction (Fig. 1(c)) is plotted as a function of applied bias voltage in Fig. 1(d), showing the expected linear relationship between the step in potential and applied bias across the junction for a reverse biased $p$ - $n$ junction. It is immediately apparent from this plot that the FIBprepared $p$ - $n$ junction specimen responds to the applied voltage, with the potential step across the junction increasing with applied reverse bias. Assuming that i) the electrically active specimen thickness is the same on both the $n$ - and the $p$ - sides of the junction ${ }^{21}$, ii) the phase shift due to the junction is contained within the specimen and iii) the applied bias is dropped fully across the junction and not elsewhere on the specimen or holder, then the slope and intercept of the graph shown in Fig. 1(d) provide values for the electrically active specimen thickness and the built-in potential across the junction of $500 \pm 10 \mathrm{~nm}$ and $0.9 \pm 0.1 \mathrm{~V}$, respectively, by using the expressions

$$
\left\{\begin{array}{l}
\Delta \varphi=4.35 \mathrm{~V}_{\mathrm{app}}+3.92 \\
\Delta \varphi=\mathrm{C}_{\mathrm{E}} \mathrm{tV}_{\mathrm{app}}+\mathrm{C}_{\mathrm{E}} \mathrm{tV}_{\mathrm{bi}}
\end{array} \Rightarrow \mathrm{t}=500 \pm 10 \mathrm{~nm}, \mathrm{~V}_{\mathrm{bi}}=0.9 \pm 0.1 \mathrm{~V}\right.
$$

where $\Delta \varphi, V_{a p p}$ and $V_{b i}$ are the phase step, applied reverse bias voltage and built-in potential across the $p$ - $n$ junction, respectively. Fig. 1(e) shows representative potential, electric field and charge density 

bias, assuming the full $550 \mathrm{~nm}$ crystalline thickness of the specimen (measured using CBED) when converting the phase images into maps of electrostatic potential $V$, electric field $E$ and charge density $\rho$ using the expressions:

$$
\begin{aligned}
& V(x, y)=\varphi(x, y) / C_{E} t \\
& E(x, y)=-\nabla \cdot V(x, y) \\
& \rho(x, y)=-\varepsilon_{\mathrm{Si}} \varepsilon_{0} \nabla^{2} V(x, y)
\end{aligned}
$$

where $\varepsilon_{\mathrm{Si}}=11.7$ and $\varepsilon_{0}=8.85 \times 10^{-12} \mathrm{~F} / \mathrm{m}$.

The difference between the crystalline specimen thickness measured using CBED and the electrically active specimen thickness inferred from the step in phase plotted as a function of applied voltage is $50 \pm 10 \mathrm{~nm}$, suggesting that there is a $25 \pm 5 \mathrm{~nm}$ crystalline layer on each surface of the specimen that is depleted due to a combination of electrical surface states (i.e., surface depletion) and FIB damage ${ }^{22}$. The thickness of the depleted and inactive crystalline surface layer has been widely assumed in the literature to be the primary explanation for low values of steps in phase obtained from electron holography results ${ }^{23-27}$.

The measured built-in potential, $0.9 \pm 0.1 \mathrm{~V}$, is just in agreement with the expected theoretical value of 1.02 V, while the slope of Fig. 1(d) can be explained by considering an electrically dead layer of thickness $50 \pm 10 \mathrm{~nm}^{28}$. However, there are other discrepancies in the experimental measurements, which are not consistent with this explanation alone. In Fig. 2, experimental electric field and charge density profiles obtained from Fig. 1(e) are compared with classical one-dimensional solutions of the Poisson equation for an abrupt symmetrical Si $p-n$ junction with the measured dopant concentration of $6 \times 10^{18} \mathrm{~cm}^{-3}$. Although the general trend in the experimental data is consistent with the simulations, with the depletion width and maximum electric field increasing with applied reverse bias, there are large differences between the magnitudes of the experimental and simulated values. The electric fields measured using electron holography are only about $15 \%$ of the simulated values, whether or not the thickness of the electrically inactive surface layer is taken into account, while the measured charge 
173 densities are more than an order of magnitude lower than the expected value of $6 \times 10^{18} \mathrm{~cm}^{-3}$. In

174 addition, the experimentally measured depletion regions are asymmetrical in the plots of $E$ and $\rho$ and 175 approximately 5 to 10 times wider than the simulated widths, with the measured charge density 176 increasing with reverse bias voltage instead of remaining constant. In contrast to reports in the 177 literature that electrical biasing can reactivate some of the dopants that have been deactivated by 178 specimen preparation (due to Joule heating) ${ }^{12}$, in the present study we measured the same charge 179 density at $0 \mathrm{~V}$ after many biasing cycles.

180 In contrast to previous reports ${ }^{29}$, the surface of the present FIB-prepared specimen is not an equipotential. The experimental phase images are shown in Fig. 3 in the form of eight-times-amplified phase contours and illustrate the presence of fringing fields in the vacuum region outside the specimen, which change with applied reverse bias. The phase shift in the vacuum region along the specimen edge, within the field of view, is greater than $9 \mathrm{rad}$ at $2 \mathrm{~V}$ reverse bias. It is important to note that the position of the fringing field is not aligned with the junction position within the specimen, but is shifted slightly towards the $p$-side of the junction.

187 The leakage of the electric field into the vacuum region has two consequences for off-axis electron holography. First, the assumption that the reference wave is not influenced by the electrostatic potential of the specimen is not strictly valid, and this perturbation needs to be taken into account in the interpretation of the recorded phase images. Second, the presence of the fringing field above and below the specimen needs to be considered. resolution, ii) fringing fields, and iii) surface charge on the determination of charge density from electron holographic phase images. 


\section{1) Limited spatial resolution}

197 One important factor that needs to be considered when calculating charge densities from electrostatic

198 potential maps that have been extracted from phase images is the smoothing of the potential distribution as a result of the finite spatial resolution of the experimental measurements. The effect of limited spatial resolution ( $14 \mathrm{~nm}$, as dictated by the size of the mask used in reconstructing the phase image) on the charge density distribution extracted from a phase image is illustrated in Fig. 4. In this figure, the theoretical potential distribution across an abrupt $\mathrm{Si} p$ - $n$ junction with a dopant concentration of $6 \times 10^{18} \mathrm{~cm}^{-3}$ is convoluted with a Gaussian point spread function (with a $14 \mathrm{~nm}$ standard deviation) and the charge density is then calculated from its second derivative. Fig. 4(a) shows calculated (theoretical) and smoothed potential profiles across the $p$ - $n$ junction for 0 and $2 \mathrm{~V}$ reverse bias. The limited spatial resolution has no effect on the measurement of the magnitude of the potential step across the junction if the measurement can be performed sufficiently far from the position of the junction. However, the potential profile becomes smoother as a result of the limited spatial resolution, resulting in a decrease in the charge density and an increase in the depletion width inferred from the second derivative of the potential profile. For example, the apparent charge density in Fig. 4(b) decreases from $6 \times 10^{18} \mathrm{~cm}^{-3}$ to $1 \times 10^{18} \mathrm{~cm}^{-3}$ at $0 \mathrm{~V}$ bias, while the depletion width increases from 20 to $70 \mathrm{~nm}$.

The effect of limited spatial resolution on the inferred charge density is not the same for different applied reverse bias voltages. For a larger reverse bias, the curvature of the potential profile is influenced less strongly by the limited spatial resolution, resulting in an apparent increase in charge density in Fig. 4(b), calculated from the second derivatives of the smoothed potential profiles, with applied reverse bias.

\section{2) Fringing fields}

Three-dimensional (3D) simulations of electrostatic potentials within and around TEM specimens containing $p-n$ junctions were carried out using the commercially available device simulator ATLAS 
221 by Silvaco ${ }^{30}$. By solving Poisson's equation, the electrostatic potential was calculated inside a 500-

222 nm-thick parallel-sided specimen containing an abrupt symmetrical $\mathrm{Si} p$ - $n$ junction for a dopant 223 concentration of $6 \times 10^{18} \mathrm{~cm}^{-3}$, as well as in a 750-nm-thick vacuum region above and below the specimen and in a 700-nm-thick vacuum region to the side of the specimen. A representative simulated 3D potential distribution is shown in Fig. 5 (a) for an applied bias of 0 V. This figure shows only half of the simulated volume, which continues along the $z$-axis on the opposite side of the $x y$ plane. The 750-nm-thick vacuum region above and below the specimen is large enough for the electrostatic potential variation to reach a value close to zero at the edge of the simulated volume. In order to apply an electrical bias in the simulations, electrical contacts were considered on the $n$ - and $p$ sides of the specimen at $\mathrm{y}=0$ and $1 \mu \mathrm{m}$ in Fig. 5 (a), respectively. When solving Poisson's equation, the difference between the normal components of the respective electric displacements was assumed to be equal to surface charge densities (Neumann boundary conditions) at the positions of the planes with no electrical contacts. At the electrical contacts, a fixed surface potential, fixed electron concentrations and fixed hole concentrations (Dirichlet boundary conditions) were used as boundary conditions. These boundary conditions for the electrical contacts were chosen because experimentally the electrical contacts are over $1 \mathrm{~mm}$ away from the region of interest and therefore the drop in voltage across the electrical contacts and its consequent fringing fields do not affect the holography observation. The boundary condition used here for the electrical contacts result in no drop in the electrostatic potential in the semiconductor close to the electrical contacts. In order to investigate the effect of fringing fields on the projected potential, the specimen surface was assumed to have a negligible surface state density in the simulations. The phase shift that the electron beam experiences as it passes through the $3 \mathrm{D}$ potential distribution was calculated by integrating the electrostatic potential along the electron beam direction (the $z$-axis in Fig. 5(a)) and then multiplying the projected potential by the constant $C_{E}$, according to Eq.1. The perturbation of the reference wave by the fringing field was also considered when calculating the simulated phase images, using an overlap width of $500 \mathrm{~nm}$ (similar to that measured experimentally). The mean inner potential of $\mathrm{Si}(\sim 12 \mathrm{~V})$ was not included in the present simulations, but should have no effect on the calculated electric field and 
charge density, since it simply adds a constant to the electrostatic potential inside the specimen relative to that in vacuum.

250

A representative simulated phase image and corresponding eight-times-amplified phase contours are shown before and after considering the perturbation of the reference wave for an applied reverse bias of $0 \mathrm{~V}$ in Figs. 5(b) and (c), respectively. The profiles in Fig. 5(d) represent phase profiles across the junction at $0 \mathrm{~V}(\mathrm{a}, \mathrm{b}$ and $\mathrm{c})$ and $2 \mathrm{~V}\left(\mathrm{a}^{\prime}, \mathrm{b}^{\prime}\right.$ and $\left.\mathrm{c}^{\prime}\right)$ reverse bias. In this figure, the profiles marked (a) and $\left(a^{\prime}\right)$ show the phase change across the junction within the specimen without considering the effects of fringing fields, those marked (b) and $\left(b^{\prime}\right)$ show the entire phase change across the junction, including the fringing fields above and below the specimen, while those marked (c) and (c') show the phase profiles after including the effect of the perturbed reference wave. The difference between profiles $\left(\mathrm{c}^{\prime}\right)$ and $\left(\mathrm{a}^{\prime}\right)$ shows how much the fringing fields and the perturbed reference wave are predicted to contribute to the phase shift of the electron beam at $2 \mathrm{~V}$ reverse bias. The phase step across the junction in this 500-nm-thick specimen is predicted to increase by approximately a factor of three when the contributions to the phase shift from fringing fields above and below the specimen and the perturbed reference wave are considered. Profiles $(b)$ and $\left(b^{\prime}\right)$ show that the presence of fringing fields above and below the specimen can introduce a difference in slope in the phase profiles between the $p$ - and $n$-side of the junction, as well as resulting in a larger phase difference between the $p$ - and $n$ side further from the junction. This difference in slope increases with applied reverse bias. By taking the perturbation of the reference wave into account (profiles (c) and (c') in Fig. 5(d)), the phase step across the junction decreases slightly. However, the profiles also become less flat and the slope of the phase profile on the $p$ - and $n$ - side changes such that further from the junction the phase difference between the $p$-side and $n$-side decreases, when compared to that measured close to the junction.

In Fig. 5(e), the calculated phase step across the junction is shown before considering the effects of fringing fields and the perturbed reference wave (black triangles), after considering the contribution to the phase shift due to fringing fields above and below the specimen but without considering the perturbed reference wave (blue circles), and after taking the effect of the perturbed reference wave into account (red squares), plotted as a function of applied reverse bias. It can be seen that the 
presence of fringing fields does not affect the linear relationship between the phase step and the applied reverse bias. Although the phase step increases linearly with applied reverse bias in all three cases, the slope and intercept of the fitted lines (shown in the figure) are different. Without considering fringing fields, the intercept of the fitted line represents the product of the specimen thickness, the built-in potential and the constant $C_{E}$ (Eq. 3). After including the contribution to the phase shift from fringing fields above and below the specimen, both the intercept and the slope of the fitted line increase. The values then decrease only slightly when perturbation of the reference wave is taken into account. An important point to note is that the fringing fields do not change the interceptto-slope ratio, which provides a measure of the built-in potential across the junction. This means that the built-in potential extracted from the plot of phase step versus applied reverse bias is not sensitive to the presence of fringing fields. This conclusion can also be reached by analytical calculations ${ }^{31}$.

In contrast to the experimental observations, when the effects of fringing fields are included in the simulations, the inferred electric fields and charge densities increase significantly when compared to calculations for no fringing fields. The same processing steps were applied to the simulations as to the experimental phase images to obtain the difference electric field and charge density distributions shown in Figs. 5(f) and (g) for different applied bias voltages. For example, the electric field profile shown in Fig. 5(f) for a $2 \mathrm{~V}$ reverse bias is the difference between the electric fields calculated from phase profiles $\left(\mathrm{c}^{\prime}\right)$ and $\left(\mathrm{a}^{\prime}\right)$. The electric fields and charge densities contributed by the fringing fields show the same trend as the electric fields and charge densities across the $p$ - $n$ junction in response to applied reverse bias, but their magnitudes are larger than those shown in Figs. 2(b) and (d). For example, the maximum electric field at $2 \mathrm{~V}$ reverse bias determined from the simulated phase image including the effects of fringing fields (profile ( $\mathrm{c}^{\prime}$ ) in Fig. 5(d)) is $3800 \mathrm{kV} / \mathrm{cm}$, which is the sum of the electric field across the junction $(1600 \mathrm{kV} / \mathrm{cm})$ at this applied voltage and the contribution from the presence of fringing fields $(2200 \mathrm{kV} / \mathrm{cm})$.

The simulations show that, if perfect surfaces with no surface states and damage are assumed for a $p-n$ junction specimen, then the contribution from fringing fields to the phase step across the junction is predicted to be larger than that caused by the $p-n$ junction within the specimen. In contrast, the 
presence of fringing fields does not have a severe effect on the measurement of the depletion width, as the depletion widths in Figs. 5(f) and (g) are approximately in agreement with those in Figs. 2(b) and (d). The built-in potential extracted from the phase step versus applied voltage plot is also not affected significantly by the fringing fields.

\section{3) Positive surface charge}

In an attempt to investigate the effect of surface states and secondary electron emission on the measurement of electrostatic potentials using off-axis electron holography, the above 3D electrostatic potential simulation was repeated for the same $p-n$ junction specimen, in the same geometry, but including a uniform positive surface charge on its surfaces.

The origin of surface states in a FIB-prepared TEM specimen could be a combination of surface termination, ion beam damage and high-energy electron beam irradiation ${ }^{28,32}$. Regardless of the origin, the overall effect of surface states in the presence of electron beam irradiation is likely to result in the presence of positive surface charge on the specimen surfaces. Since few primary electrons are absorbed by a TEM specimen when compared with the number of emitted secondary electrons, it is expected that in the absence of good electrical conductivity on the specimen surfaces they will charge positively $^{33,34}$. It is difficult to measure the surface charge density independently. However, we assume a positive surface charge density of $8 \times 10^{12}$ e.c. (electron charges) $/ \mathrm{cm}^{2}$ on all three surfaces of the specimen (top, bottom and edges) in our simulation, based on a comparison between simulated phase profiles in the vacuum region and our experimental electron holographic phase images. Simulated phase profiles for different uniform surface charge densities between $1 \times 10^{12}$ and $1 \times 10^{13}$ e.c. $/ \mathrm{cm}^{2}$ in intervals of $1 \times 10^{12}$ e.c. $/ \mathrm{cm}^{2}$ were compared with a corresponding phase profile taken along the specimen edge in the vacuum region from the experimental phase image recorded at $0 \mathrm{~V}$ bias. The closest match between the experimental and simulated phase profile in the vacuum region at $0 \mathrm{~V}$ bias was obtained for a surface charge density of $8 \times 10^{12}$ e.c. $/ \mathrm{cm}^{2}$. For higher surface charge densities, the fringing fields disappeared completely, while for lower surface charge densities much stronger fringing fields than those measured experimentally appeared in the vacuum region. As a result of 
assuming a positive surface charge density of $8 \times 10^{12}$ e.c. $/ \mathrm{cm}^{2}$, the specimen surfaces on the $p$-side became inverted to have $n$-type character. Assuming the depletion approximation ${ }^{32}$, such a charge density would result in a surface depletion width of approximately $11 \mathrm{~nm}$ and a surface potential difference of approximately $0.6 \mathrm{~V}$ on the $p$-side of the specimen, far from the junction. The strong inversion on the $p$-side, when the electron concentration at the surface is equal to the dopant concentration in the bulk ${ }^{32}$, occurs in this specimen if a positive surface charge density of approximately $10^{18}$ e.c. $/ \mathrm{cm}^{2}$ is assumed on the specimen surface, resulting in a maximum surface depletion width of approximately $15 \mathrm{~nm}^{35}$. For the purpose of the simulations, the surface charge was considered to be embedded in a $2 \mathrm{~nm}$ oxide layer on the specimen surface. The 3D potential distribution obtained from such a simulation is shown in Fig. 6(a). The phase image determined from the simulated potential distribution, as well as corresponding eight-times-amplified phase contours, are shown in Fig. 6(b), taking into account the perturbed reference wave. From Figs. 6(a) and 5(a), it can be seen that the presence of positive surface charge decreases the leakage of electric fields into the vacuum region, with the electrostatic potential variation in the vacuum region in Fig. 6(a) now limited to the proximity of the specimen surfaces when compared to Fig. 5(a). The fringing fields are not only weaker, as can be seen in the eight-times-amplified phase contours shown in Fig. 6(b), but they are also not aligned with the junction position within the specimen in the presence of surface charge.

The calculated phase step across the junction in the presence of surface charge is plotted in Fig. 6(c) as a function of applied reverse bias. The phase steps marked with red squares are calculated from the entire simulated volume, whereas the phase steps marked with green diamonds and blue triangles show the contributions from the fringing fields in the vacuum region and the potential variation within the specimen, respectively. At $0 \mathrm{~V}$ bias, the calculated phase step associated with the potential variation within the specimen is larger than that from the fringing fields, while at $2 \mathrm{~V}$ reverse bias the opposite is the case.

Figs. 6(d) and (e) show electric fields and charge densities, respectively, calculated from the phase shift caused only by fringing fields, as in Figs. 5(f) and (g). The electric fields and charge densities calculated from the phase shift determined from the entire simulated volume are shown in Figs. 6(f) 
and $(\mathrm{g})$, respectively. The electric fields and charge densities caused by the fringing fields alone are significantly smaller now that positive charge is included on the specimen surface. Both the electric field and the charge density are asymmetrical in the presence of surface charge, with a lower inferred charge density on the $p$-side than on the $n$-side. The inferred charge density increases with applied reverse bias in the presence of surface charge, whereas it does not change with applied bias if no surface charge is included (Fig. $5(\mathrm{~g})$ ). The depletion width is also wider in the presence of surface charge. For example, in Fig. 6(g), the depletion width is approximately $80 \mathrm{~nm}$ at $2 \mathrm{~V}$ reverse bias, whereas in the absence of surface charge it is below $50 \mathrm{~nm}$ (Figs. 2(b) and $5(\mathrm{~g})$ ).

\section{4) Discussion and Summary}

In the experimental section of this paper, it was shown that a Si $p-n$ junction specimen prepared using FIB milling responds to an applied electrical bias. In qualitative agreement with theory, the potential step, electric field and depletion width across the junction, measured from electron holographic phase images, increase with applied reverse bias. However, instead of remaining constant, the measured charge density increases with applied reverse bias. In contrast to previous reports, but in agreement with theory, fringing fields are observed in the vacuum region close to the specimen edge. The fringing fields increase in magnitude with applied reverse bias.

Quantitative comparisons between the experimental results and classical one-dimensional solutions of the Poisson equation for an abrupt $\mathrm{Si} p-n$ junction reveal more discrepancies than agreement. Although the built-in potential determined from a plot of phase step versus applied reverse bias is approximately in agreement with the value expected from theory, the electrically active specimen thickness determined from this plot is $50 \mathrm{~nm}$ smaller than the crystalline thickness of the specimen measured using CBED. Although one can explain this discrepancy by assuming an electrically inactive crystalline layer on the top and bottom surfaces of the specimen, the presence of fringing fields means that they need to be considered to interpret the measured phase step across the junction. More significantly, the measured electric fields and charge densities are $85 \%$ and an order of magnitude smaller than the expected values, respectively, while the measured depletion widths are too 
high by $\sim 300 \%$, the measured charge density is asymmetrical and the fringing fields are not aligned with the position of the junction within the specimen. These discrepancies cannot be explained by the assumption of a simple electrically inactive layer on the top and bottom surfaces of the specimen.

In the simulation section, we investigated the effects of limited spatial resolution, fringing fields and surface charge on the electron holography measurements. Simulations presented in this paper did not account for all of the discrepancies, particularly the large depletion width measured experimentally. Limited spatial resolution smooths the potential distribution and results in a lower electric field, a lower charge density and a larger depletion width determined from the projected potential profile. As the effect of limited spatial resolution on the potential step across a $p$ - $n$ junction is smaller for a larger applied reverse bias, a larger charge density is then inferred. Limited spatial resolution is likely to be part of the explanation for the low values of measured electric field and charge density and the large values of depletion width, both in the present study and in other reports ${ }^{16}$. However, neither the full extent of the discrepancies nor the asymmetrical charge density profiles can be explained by limited spatial resolution alone. For studying modern nanoscale devices, which was not the aim of this work, a large field of view is not necessary and therefore spatial resolution is not a limiting factor.

If the specimen surfaces are assumed to be ideal, with negligible surface states and defects, then electric fields are predicted to leak out from the $p$ - $n$ junction into vacuum and to generate strong fringing fields that can affect the phase image significantly. Our simulations show that the phase shift caused by fringing fields can then be about two times larger than that caused by the potential variation inside a 500-nm-thick specimen containing a symmetrical abrupt $\mathrm{Si} p$-n junction with a dopant concentration of $6 \times 10^{18} \mathrm{~cm}^{-3}$. When calculating electric field and charge density distributions from phase images, the contribution from the phase shift caused by the fringing fields can then be larger than that originating from the interior of the specimen. However, the determination of the built-in potential from the intercept to slope ratio of a plot of phase step versus applied reverse bias is not affected significantly by the fringing fields in the absence of surface charges. When compared with this simulation, significantly weaker fringing fields are observed experimentally, suggesting that the surfaces of TEM specimens in the presence of electron irradiation cannot be assumed to have 
negligible surface state concentrations. In modern devices, in which dopant concentrations can reach a

409 few percent, the fringing fields are expected to be stronger.

410 Simulations incorporating positively charged specimen surfaces were used to model the effects of secondary electron emission during electron irradiation. In order to reproduce the phase shift in the

412 vacuum region close to the specimen edge measured experimentally at $0 \mathrm{~V}$ bias, a uniform positive surface charge of $8 \times 10^{12}$ e.c. $/ \mathrm{cm}^{2}$ had to be included on the specimen surfaces in the simulation. When compared with simulations for ideal specimen surfaces, the presence of surface charges resulted in weaker fringing fields, lower electric fields, smaller charge densities and wider depletion widths. Moreover, the calculated electric fields and charge densities in the presence of surface charges were asymmetrical, the inferred charge densities increased with applied reverse bias and the fringing fields in vacuum close to the specimen edge were shifted slightly. These observations are all in qualitative agreement with the experimental measurements, suggesting that the presence of positive surface charge on the TEM specimen surface is one of the reasons behind the discrepancies seen between our experimental results and initial simulations.

The quality of FIB-prepared surfaces directly affects the strength of fringing fields and is likely to be the reason for the absence of fringing fields in previous studies. It is therefore necessary to develop a standard FIB-based specimen preparation recipe that provides reproducible surfaces and a corresponding electrostatic potential model that predicts the effect of such surface conditions on the electrostatic potential distribution inside and outside the specimen.

In conclusion, the discrepancies between experiment and theory seen in electron holographic studies of semiconductor devices are likely to have four different origins: 1) the failure of the dopant potential model used for bulk samples in a thin specimen in which surface termination plays a role; 2) changes in the potential distribution during specimen preparation associated with surface damage and implantation, 3) alteration of the original potential distribution as a result of high-energy electron

432 beam irradiation, which results in secondary electron emission, electron-hole pair and point defect generation and 4) other sources of error such as dynamical diffraction and limited spatial resolution. 
434 Further studies are in progress in our group ${ }^{36}$ and by others ${ }^{17,37-40}$ to disentangle the role and 435 contribution of these parameters in electron holographic studies of semiconductor devices. Attempts 436 are also being made to electrically bias more complex device structures in situ in the TEM to measure 437 electrostatic potential distributions under working conditions ${ }^{41-46}$.

\section{Acknowledgements}

439 We are grateful to C. B. Boothroyd, B. E. Kardynal, P. A. Midgley, R. S. Pennington and G. Pozzi for

440 valuable discussions. Financial support is gratefully acknowledged from the EPSRC through a 441 Science and Innovation award. 
${ }^{1}$ T. Shinada, S. Okamoto, T. Kobayashi, and I. Ohdomari, Nature 437, 1128 (2005).

$444 \quad{ }^{2}$ International Technology Roadmap for Semiconductors,

445 http://www.itrs.net/links/2010ITRS/home2010.htm (2010).

$446 \quad{ }^{3}$ D. Cooper, J.-L. Rouviere, A. Béché, S. Kadkhodazadeh, E.S. Semenova, K. Yvind, and R.E.

447 Dunin-Borkowski, Appl. Phys. Lett. 99, 261911 (2011).

$448{ }^{4}$ A.C. Twitchett-Harrison, T.J. V Yates, R.E. Dunin-Borkowski, and P.A. Midgley, Ultramicroscopy 449 108, 1401 (2008).

$450 \quad{ }^{5}$ H. Lichte and M. Lehmann, Rep. Prog. Phys. 71, 016102 (2008).

$451 \quad{ }^{6}$ D. Cooper, R. Truche, P. Rivallin, J.-M. Hartmann, F. Laugier, F. Bertin, A. Chabli, and J.-L.

452 Rouviere, Appl. Phys. Lett. 91, 143501 (2007).

$453{ }^{7}$ D. Cooper, A.C. Twitchett-Harrison, P.A. Midgley, and R.E. Dunin-Borkowski, J. Appl. Phys. 101, $454094508(2007)$.

$455{ }^{8}$ M.-G. Han, Y. Zhu, K. Sasaki, T. Kato, C.A.J. Fisher, and T. Hirayama, Solid State Electron. 54, 456777 (2010).

$457{ }^{9}$ A.C. Twitchett-Harrison, Electron Holography of Semiconductor Devices, PhD thesis, University of 458 Cambridge, 2002.

$459 \quad{ }^{10}$ S. Frabboni, G. Matteucci, G. Pozzi, and M. Vanzi, Phys. Rev. Lett. 55, (1985).

$460 \quad{ }^{11}$ M.-G. Han, D.J. Smith, and M.R. McCartney, Appl. Phys. Lett. 92, 143502 (2008).

$461{ }^{12}$ A.C. Twitchett-Harrison, R.E. Dunin-Borkowski, R.F. Broom, and P.A. Midgley, J. Phys. Condens. 462 Matter 16, S181 (2004).

$463 \quad{ }^{13}$ S. Frabboni, G. Matteucci, and G. Pozzi, Ultramicroscopy 23, 29 (1987).

$464{ }^{14}$ A.C. Twitchett-Harrison, R.E. Dunin-Borkowski, and P.A. Midgley, Phys. Rev. Lett. 88, 238302 465 (2002).

$466{ }^{15}$ A.C. Twitchett-Harrison, R.E. Dunin-Borkowski, and P.A. Midgley, Scanning 30, 299 (2008).

$467{ }^{16}$ A.C. Twitchett, R.E. Dunin-Borkowski, R.J. Hallifax, R.F. Broom, and P.A. Midgley, Microsc. 468 Microanal. 11, 1 (2005).

$469{ }^{17}$ H. Lichte, F. Börrnert, A. Lenk, A. Lubk, F. Röder, J. Sickmann, S. Sturm, K. Vogel, and D. Wolf, 470 Ultramicroscopy 134, 126 (2013).

$471 \quad{ }^{18}$ J.B. Park, T. Niermann, D. Berger, A. Knauer, I. Koslow, M. Weyers, M. Kneissl, and M.

472 Lehmann, Appl. Phys. Lett. 105, 094102 (2014).

$473 \quad{ }^{19}$ http://www.okmetic.com. 
${ }^{20}$ T. Kasama, R.E. Dunin-Borkowski, L. Matsuya, R.F. Broom, A.C. Twitchett-Harrison, P.A. Midgley, S.B. Newcomb, A.C. Robins, D.W. Smith, J.J. Gronsky, C.A. Thomas, and P.E. Fischione, in Mater. Res. Soc. Symp. Proc. (2006), pp. 2-7.

${ }^{21}$ A.C. Twitchett-Harrison, T.J. V. Yates, S.B. Newcomb, R.E. Dunin-Borkowski, and P.A. Midgley, Nano Lett. 7, 2020 (2007).

${ }^{22}$ D. Cooper, C. Ailliot, R. Truche, J.-P. Barnes, J.-M. Hartmann, and F. Bertin, J. Appl. Phys. 104, 064513 (2008).

${ }^{23}$ W.D. Rau, P. Schwander, F.H. Baumann, W. Höppner, and A. Ourmazd, Phys. Rev. Lett. 82, 2614 (1999).

${ }^{24}$ M.R. McCartney, M.A. Gribelyuk, J. Li, P. Ronsheim, J.S. McMurray, and D.J. Smith, Appl. Phys. Lett. 80, 3213 (2002).

${ }^{25}$ Z. Wang, T. Kato, N. Shibata, T. Hirayama, N. Kato, K. Sasaki, and H. Saka, Appl. Phys. Lett. 81, 478 (2002).

${ }^{26}$ Z.H. Wu, M. Stevens, F.A. Ponce, W. Lee, J.H. Ryou, D. Yoo, and R.D. Dupuis, Appl. Phys. Lett. 90, 032101 (2007).

${ }^{27}$ N. Ikarashi, H. Takeda, K. Yako, and M. Hane, Appl. Phys. Lett. 100, 143508 (2012).

${ }^{28}$ D. Cooper, C. Ailliot, J. Barnes, J. Hartmann, P. Salles, G. Benassayag, and R.E. Dunin-Borkowski, Ultramicroscopy 110, 383 (2010).

${ }^{29}$ D. Cooper, P. Rivallin, J. Hartmann, A. Chabli, and R.E. Dunin-Borkowski, J. Appl. Phys. 106, 064506 (2009).

${ }^{30} \mathrm{http}: / / \mathrm{www}$. silvaco.com.

${ }^{31}$ P.F. Fazzini, G. Pozzi, and M. Beleggia, Ultramicroscopy 104, 193 (2005).

${ }^{32}$ S.M. Sze, Physics of Semiconductor Devices (John Wiley \& Sons, 1981).

${ }^{33}$ M. Beleggia, P.F. Fazzini, P.G. Merli, and G. Pozzi, Phys. Rev. B 67, 045328 (2003).

${ }^{34}$ R.F. Egerton, P. Li, and M. Malac, Micron 35, 399 (2004).

${ }^{35}$ P.K. Somodi, A.C. Twitchett-Harrison, P.A. Midgley, B.E. Kardynal, C.H.W. Barnes, and R.E. Dunin-Borkowski, Ultramicroscopy 134, 160 (2013).

${ }^{36}$ S. Yazdi, T. Kasama, R. Ciechonski, O. Kryliouk, and J.B. Wagner, J. Phys. Conf. Ser. 471, 012041 (2013).

${ }^{37}$ D. Wolf, A. Lubk, F. Röder, and H. Lichte, Curr. Opin. Solid State Mater. Sci. 17, 126 (2013).

${ }^{38}$ A. Lenk, U. Muehle, and H. Lichte, in Microsc. Semicond. Mater. (Springer, Berlin Heidelberg, 2005), pp. 213-216.

${ }^{39}$ D. Cooper, P. Rivallin, G. Guegan, C. Plantier, E. Robin, F. Guyot, and I. Constant, Semicond. Sci. Technol. 28, 125013 (2013). 
${ }^{40}$ A. Pantzer, A. Vakahy, Z. Eliyahou, G. Levi, D. Horvitz, and A. Kohn, Ultramicroscopy 138, 36 509 (2014).

$510 \quad{ }^{41}$ S. Yazdi, T. Kasama, M. Beleggia, R. Ciechonski, O. Kryliouk, and J.B. Wagner, Microsc.

511 Microanal. 19, 1502 (2013).

$512 \quad{ }^{42}$ S. Yazdi, T. Kasama, D.W. McComb, A.C. Harrison, and R.E. Dunin-Borkowski, Microsc.

513 Microanal. 19, 1358 (2013).

$514{ }^{43}$ L.Z.-Y. Liu, C. McAleese, D. V. Sridhara Rao, M.J. Kappers, and C.J. Humphreys, Phys. Status 515 Solidi 9, 704 (2012).

$516{ }^{44}$ G. Busatto, F. Iannuzzo, D.J. Smith, D.A. Cullen, L. Zhou, and M.R. McCartney, Microelectron. 517 Reliab. 50, 1514 (2010).

$518 \quad{ }^{45}$ K. He and J. Cumings, Nano Lett. 13, 4815 (2013).

$519{ }^{46}$ K. He, J.-H. Cho, Y. Jung, S.T. Picraux, and J. Cumings, Nanotechnology 24, 115703 (2013). 

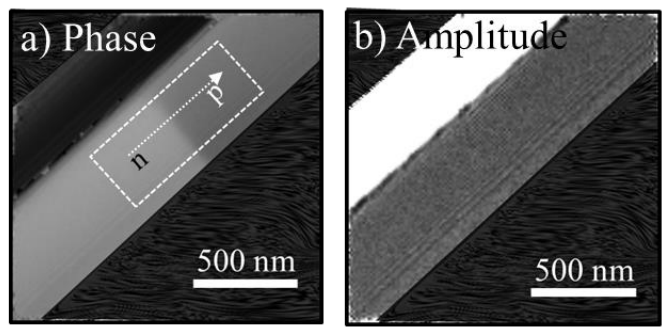

e)

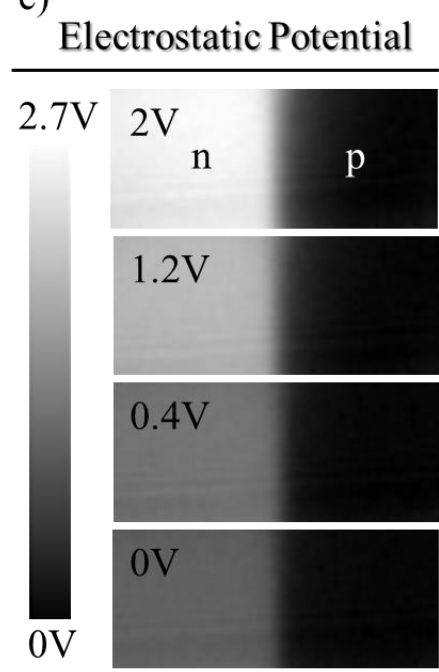

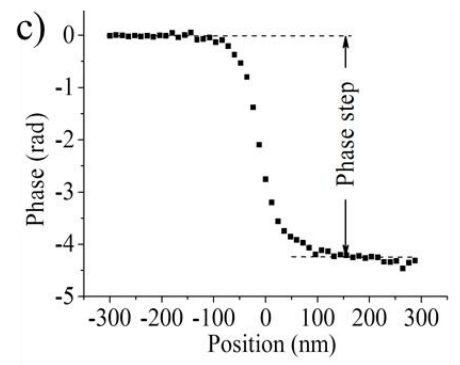

Position (nm)

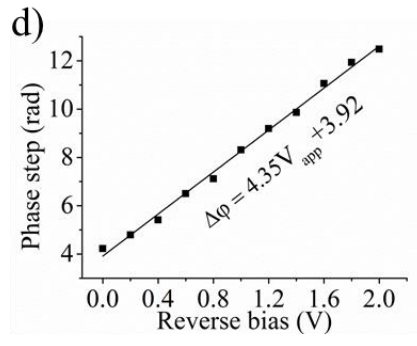

Charge density

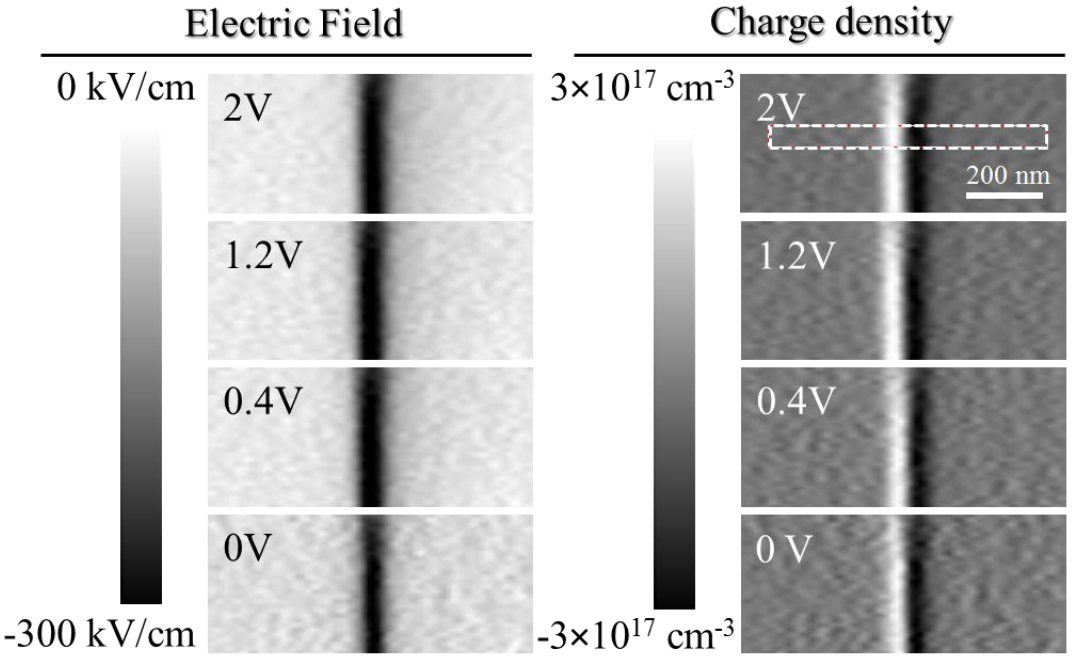

Figure 1. Representative a) unwrapped phase and b) amplitude images of the Si $p$ - $n$ junction studied here at $0 \mathrm{~V}$ applied bias. c) Phase profile measured along the arrow shown in (a). d) Phase step across the $p-n$ junction plotted as a function of applied reverse bias. The dashed box in the phase image shows the area from which e) the potential, electric field and charge density distributions were generated. Only part of the field of view is shown in each frame. The 50-nm-wide box in (e) shows the area from which the profiles in Figs. 2(a) and (c) were obtained. 

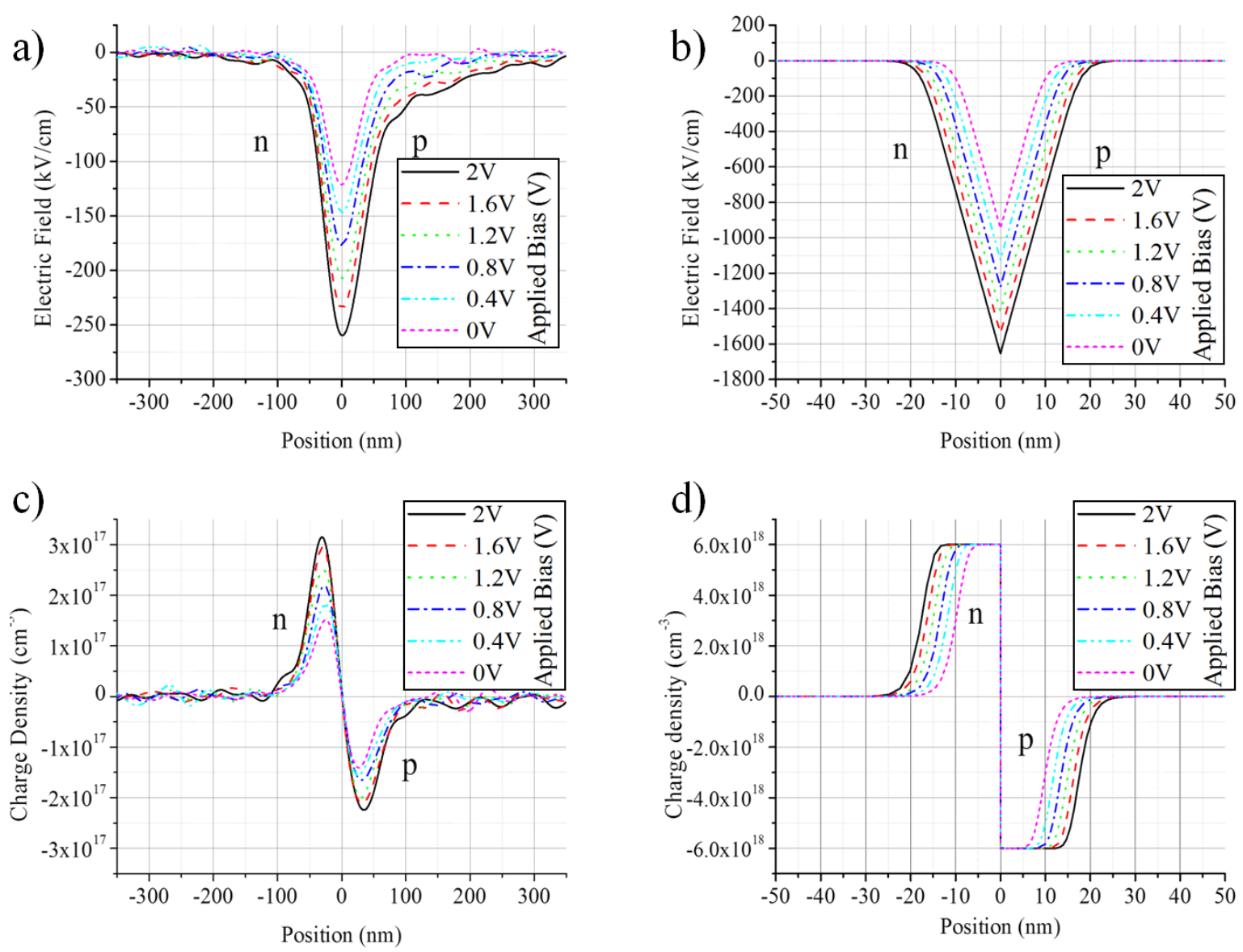

Figure 2. Measured a) electric field and c) charge density profiles across the Si $p$ - $n$ junction studied here, determined from the plots shown in Fig. 1(e) for different applied reverse bias voltages. b) and d) show corresponding simulated electric field and charge density profiles, respectively, for an abrupt symmetrical Si $p$-n junction with a dopant concentration of $6 \times 10^{18} \mathrm{~cm}^{-3}$, obtained using a onedimensional Poisson solver for the dopant species studied experimentally. The simulations are also shown as a function of applied reverse bias voltage. 


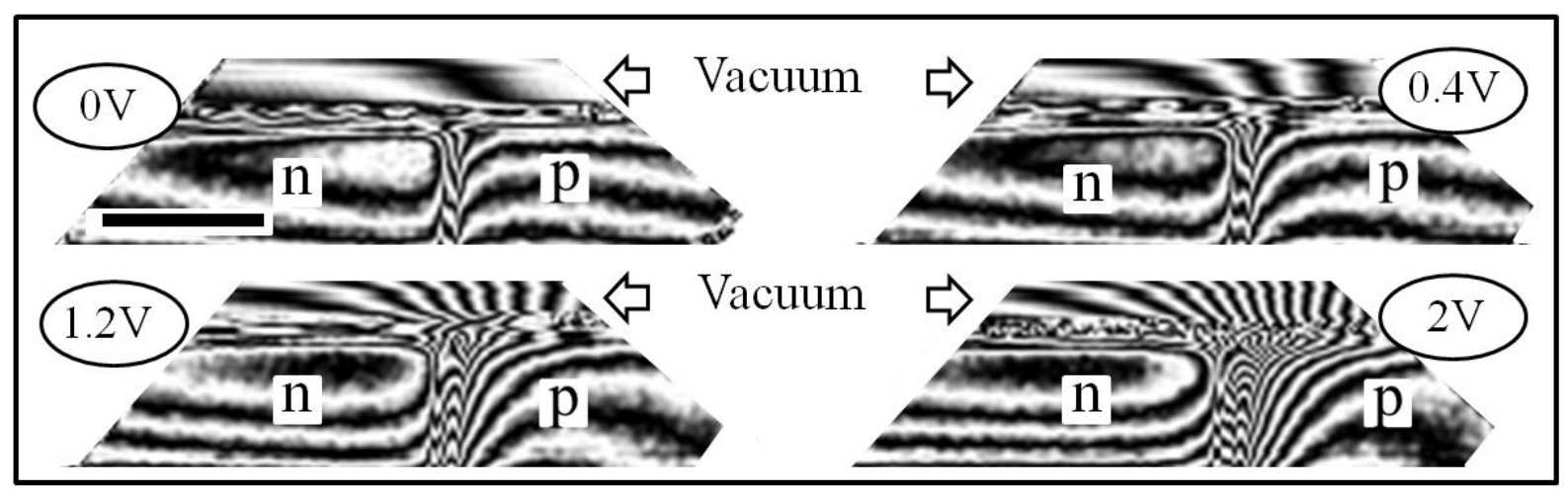

Figure 3. Eight-times-amplified phase contours shown as a function of reverse bias voltage for the Si $p$ - $n$ junction studied here. The scale bar is $500 \mathrm{~nm}$. The details of the phase contours within the depletion region should be discounted, as they contain artefacts resulting from phase amplification. In order to reduce the noise in the amplified phase contours, the phase images were smoothed. 

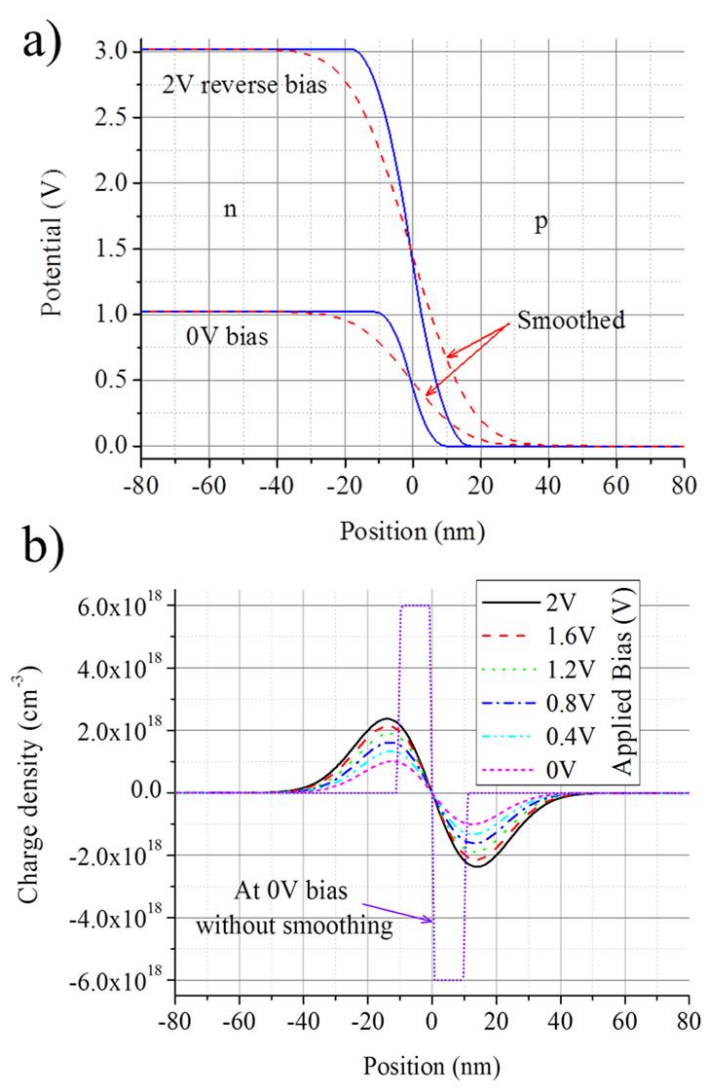

Figure 4. Illustration of the effect of limited spatial resolution on the apparent charge density determined from a potential profile for different applied reverse bias voltages, determined from simulations for an abrupt symmetrical Si $p-n$ junction with a dopant concentration of $6 \times 10^{18} \mathrm{~cm}^{-3}$. In (a) the potential profiles across the junction at $0 \mathrm{~V}$ and $2 \mathrm{~V}$ reverse bias (blue solid curves) are compared with corresponding potential profiles that were convoluted with a Gaussian point-spreadfunction with a standard deviation of $14 \mathrm{~nm}$ (red dashed curves), showing the smoothing effect of limited spatial resolution on a potential profile at two different biasing voltages. The purple dotted curve in (b) shows the charge density determined from the potential profile at $0 \mathrm{~V}$ without considering the effect of limited spatial resolution. The other curves correspond to charge densities determined from potential profiles that have been convoluted with the Gaussian point-spread-function. Note the increase in the apparent depletion width, the decrease in the apparent charge density and the variation of maximum charge density with applied reverse bias, resulting from the effects of limited spatial resolution alone. 

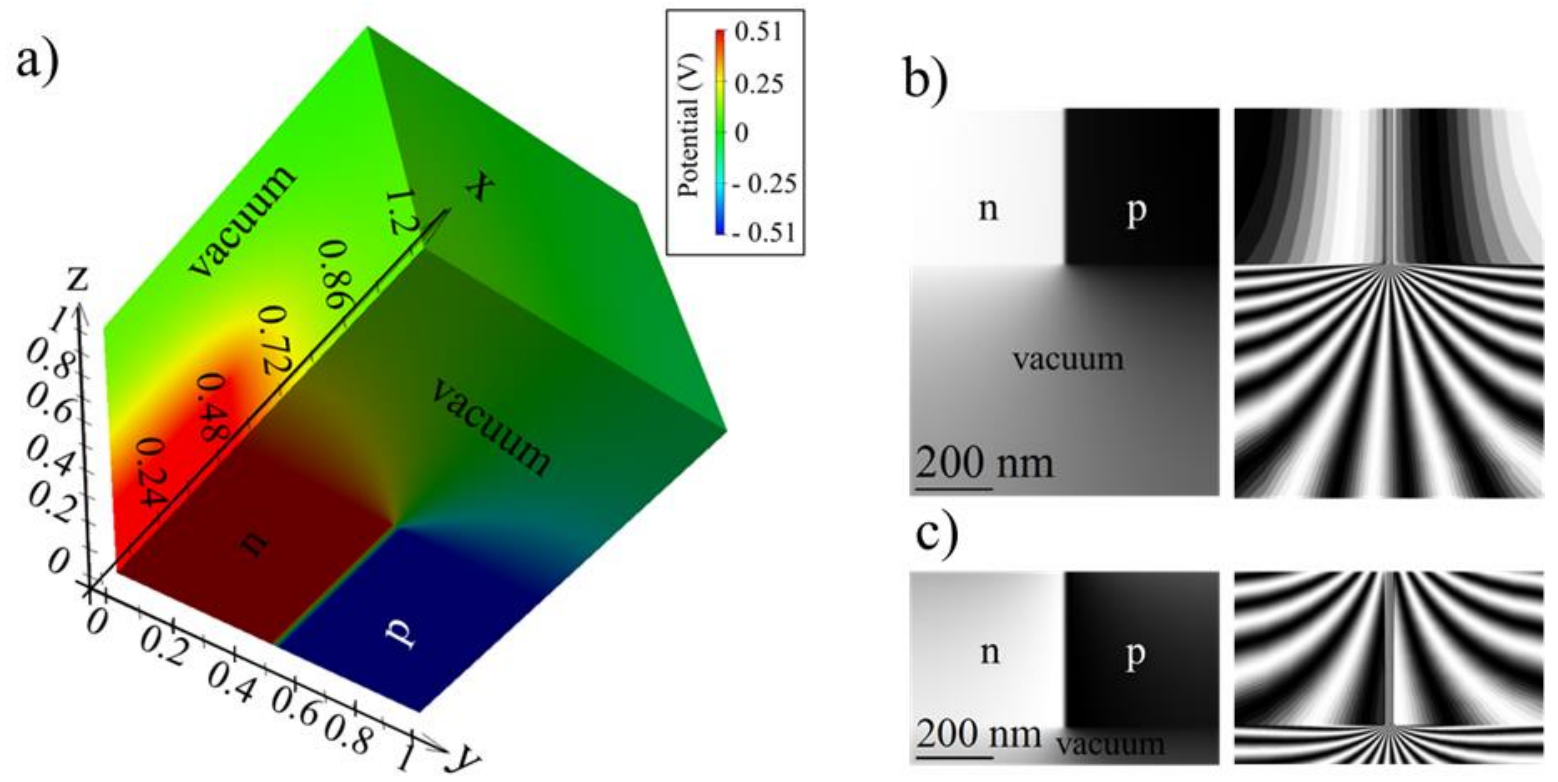

c)

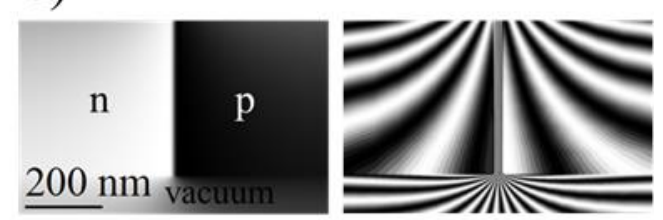

d)

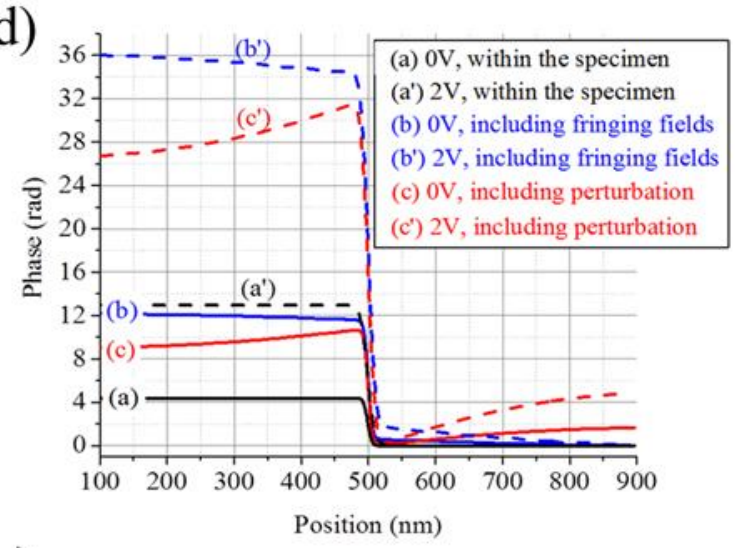

e)

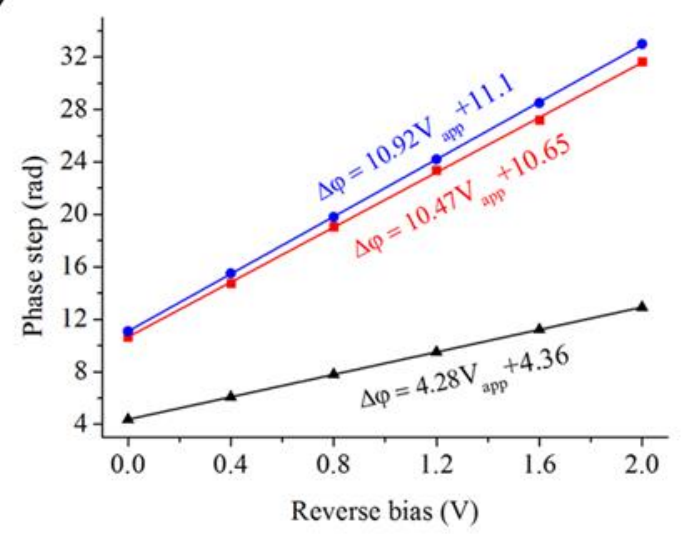

f)
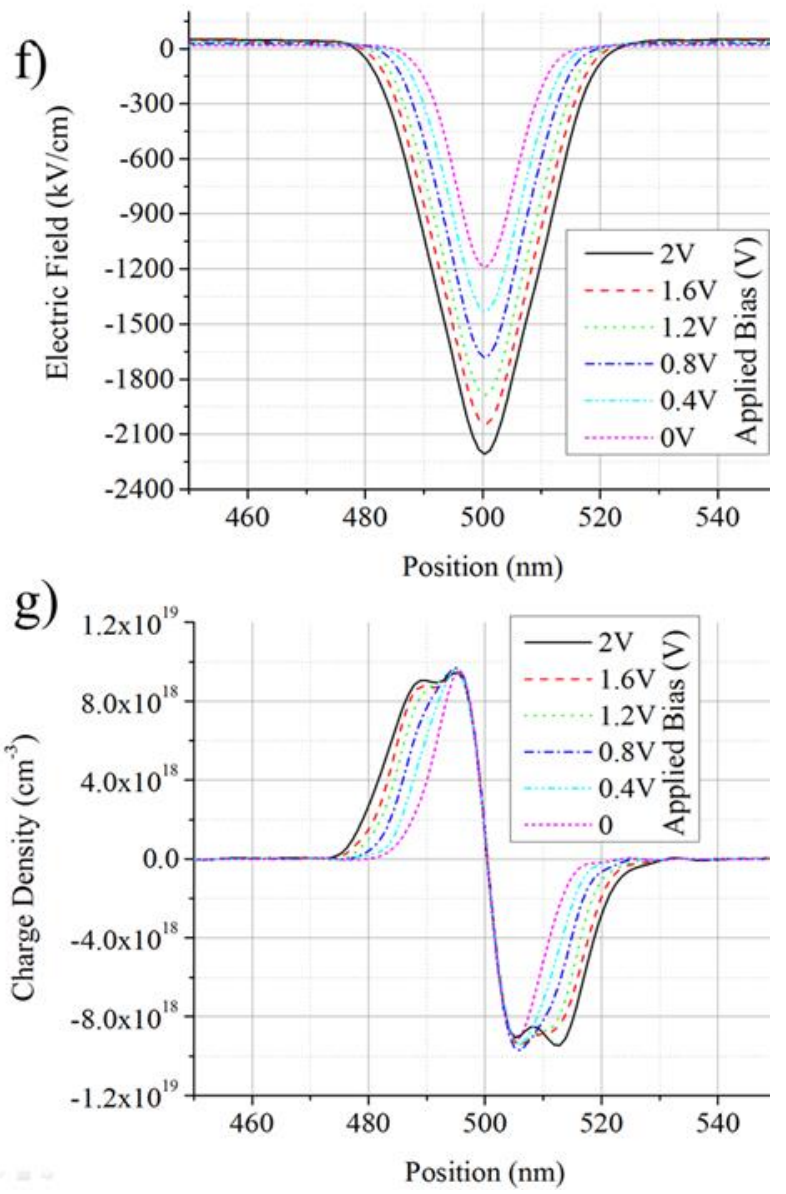

Figure 5. a) Representative 3D simulation of the electrostatic potential inside and around a 500-nmthick parallel-sided specimen containing an abrupt symmetrical Si $p$ - $n$ junction for an applied bias of $0 \mathrm{~V}$. Only half of the simulated volume (cut at the $x y$ plane) is shown, so that the potential variation within and around the specimen can be seen. A corresponding phase image, limited to a volume of 
$\mathrm{x}=0.1$ to $\mathrm{x}=1.1 \mu \mathrm{m}, \mathrm{y}=0.1$ to $\mathrm{y}=0.9 \mu \mathrm{m}$ and $\mathrm{z}=-1$ to $1 \mu \mathrm{m}$ in (a), and its eight-times-amplified phase contours calculated for an accelerating voltage of $120 \mathrm{kV}$ at $0 \mathrm{~V}$ bias, are shown in b) and c). The effect of the perturbed reference wave is taken into account in (c), but not in (b). The profiles in (d) show the phase change across the junction at $0 \mathrm{~V}(\mathrm{a}, \mathrm{b}$ and $\mathrm{c})$ and $2 \mathrm{~V}\left(\mathrm{a}^{\prime}, \mathrm{b}^{\prime}\right.$ and $\left.\mathrm{c}^{\prime}\right)$ reverse bias. The profiles marked (a) and (a') show the phase change across the junction within the specimen without considering the effects of fringing fields; those marked (b) and $\left(b^{\prime}\right)$ show the entire phase change across the junction, including the effects of fringing fields above and below the specimen; those marked (c) and ( $\left.c^{\prime}\right)$ include the effect of the perturbed reference wave. e) Contributions to the measured phase step across the junction plotted as a function of applied reverse bias from the interior of the specimen (black triangles), from the entire simulated volume without considering the perturbed reference wave (blue circles) and from the entire simulated volume taking the perturbed reference wave into account (red squares). f) and g) show electric field and charge density profiles for different applied bias voltages determined from the contribution to the phase shift from fringing fields alone. Similar to the experimental measurements shown in Fig. 1, all of the profiles are taken $300 \mathrm{~nm}$ away from the sample edge. 


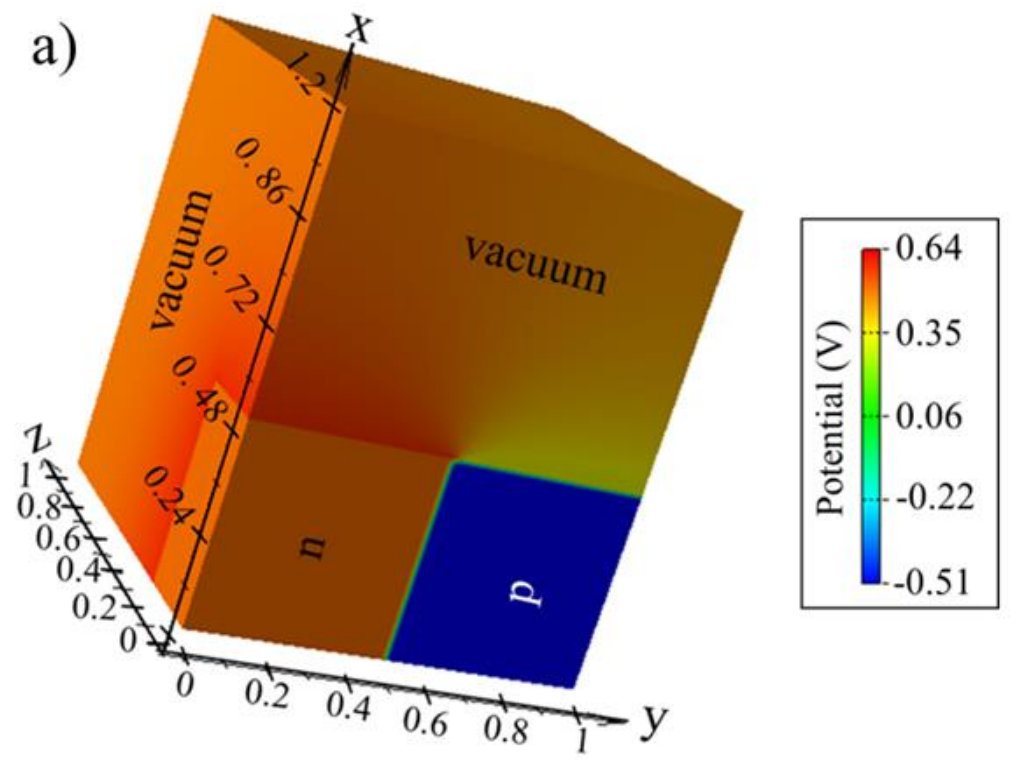

b)

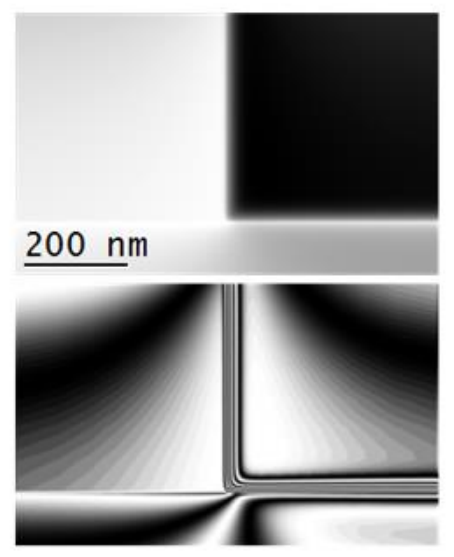

d)

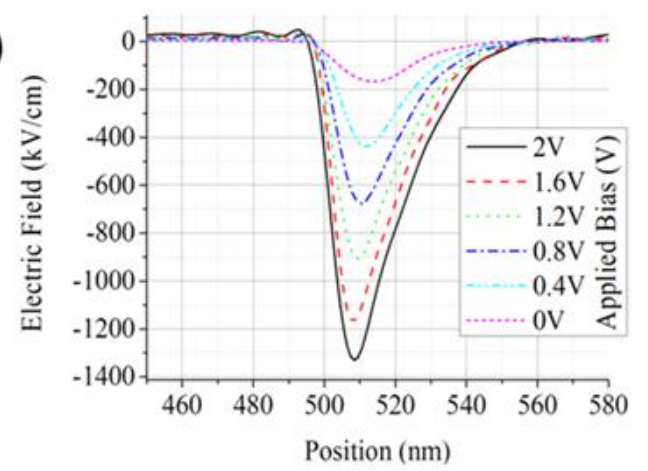

f)

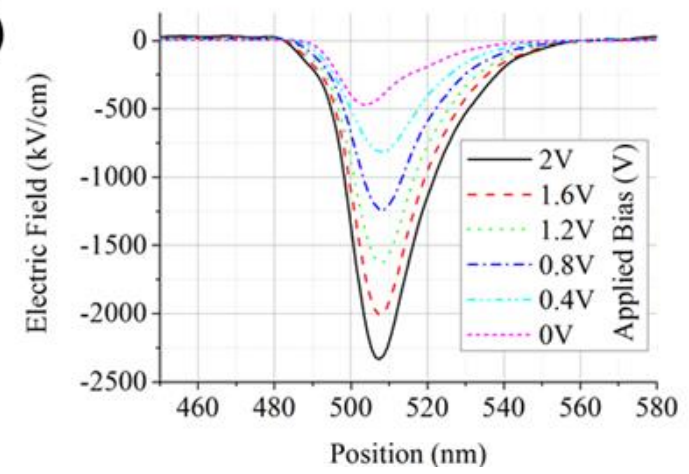

c)

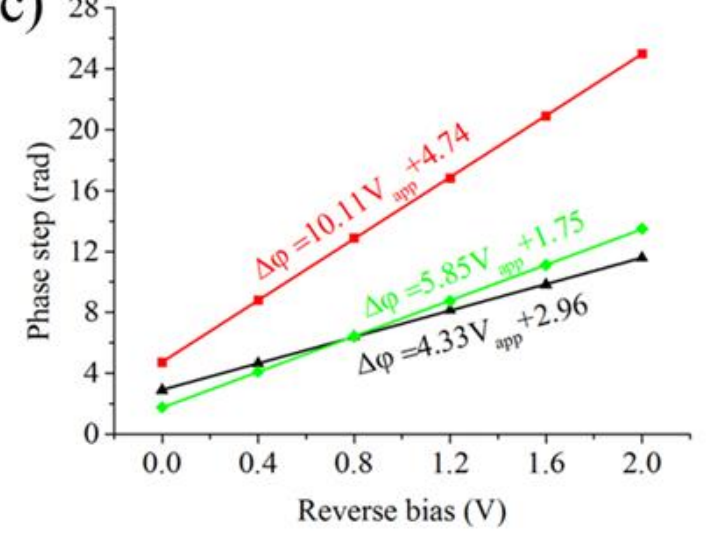

e)

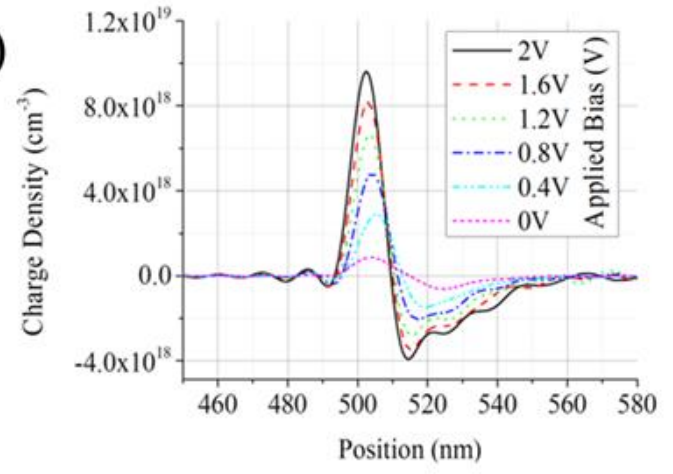

g)

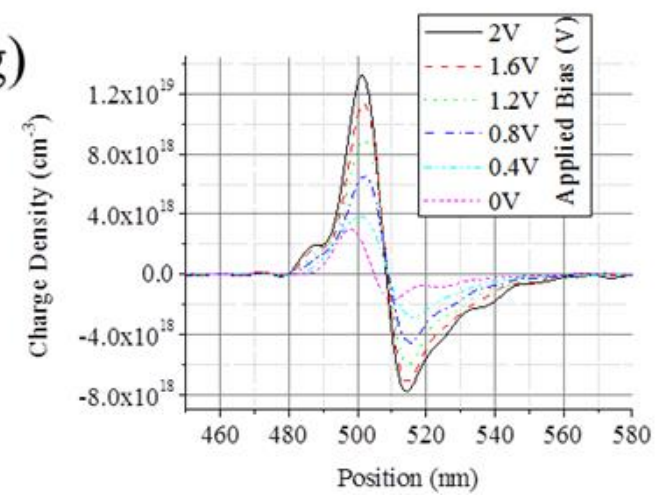


Figure 6. a) Representative 3D simulation of the electrostatic potential inside and around a 500-nmthick parallel-sided specimen containing an abrupt symmetrical Si $p$ - $n$ junction, calculated for an applied reverse bias of $0 \mathrm{~V}$ with a positive surface charge density of $8 \times 10^{12}$ e.c. $/ \mathrm{cm}^{2}$ on all three surfaces of the specimen $(\mathrm{x}=0.5 \mu \mathrm{m}, \mathrm{z}=-0.25 \mu \mathrm{m}$ and $\mathrm{z}=0.25 \mu \mathrm{m})$. b) Corresponding phase image and its eight-times-amplified phase contours. c) Contributions to the phase step across the junction measured only from the interior of the specimen (black triangles), from the entire simulated volume (red squares) and only from the fringing fields above and below the specimen (green diamonds). d) and e) show electric field and charge density profiles for different applied bias voltages determined from the phase profiles corresponding solely to contributions from fringing fields. f) and g) show electric field and charge density profiles for different applied bias voltages, determined from phase profiles corresponding to the entire simulated potential volume. All profiles are taken $300 \mathrm{~nm}$ away from the sample edge. 